\title{
EXPRESSION OF FLJB:Z66 ON A LINEAR PLASMID OF SALMONELLA ENTERICA SEROVAR TYPHI IS DEPENDENT ON FLIA AND FLHDC AND REGULATED BY OMPR
}

\author{
Shungao Xu, Xin Zou, Xiumei Sheng, Haifang Zhang, Lingxiang Mao, Hong Du, Huaxi Xu, Xinxiang Huang*
}

Department of Biochemistry and Molecular Biology, Jiangsu University School of Medical Technology, Jiangsu Zhenjiang, 212013, China.

Submitted: August 23, 2009; Returned to authors for corrections: October 09, 2009; Approved: March 16, 2010.

\begin{abstract}
Salmonella enterica serovar Typhi z66-positive strains have two different flagellin genes, fliC:d/j and $f l j B: z 66$, located on the chromosome and on a linear plasmid, respectively. To investigate the mechanism underlying the expressional regulation of $f l j B: z 66$, gene deletion mutants of the regulators FliA, FlhDC, and OmpR were constructed in this study. The expression levels of $f l i C$ and $f l j B: z 66$ were analyzed by qRT-PCR in the wild-type strain and mutants at high and low osmolarity. The results show that the expression levels of both $f l j B: z 66$ and $f l i C$ were greatly reduced in $f l i A$ and $f l h D C$ mutants under both high and low osmotic conditions. In the ompR mutant, the expression levels of fljB:z66, fliC, fliA, and $f l h D$ were increased at low osmotic conditions. SDS-PAGE and western blotting analysis of the secreted proteins revealed that the FljB:z66 was almost absent in the fliA and flhDC mutants at both high and low osmolarity. In the wild-type strain, the $f l j B: z 66$ was more highly expressed under high-osmolarity conditions than under low-osmolarity conditions. However, this difference in expression disappeared in the $\operatorname{ompR}$ mutant. Translational expression assay of FljB:z66 showed that the FljB:z66 expression was decreased in ompR mutant at both low and high osmolarity. These results suggest that the expression of $f l j B: z 66$ in S. enterica serovar Typhi is dependent on FliA and FlihDC, and OmpR can regulate the expression and secretion of FljB:z66 in different osmolarity.
\end{abstract}

Key words: Salmonella enterica serovar Typhi; fljB:z66; flhDC; fliA; ompR.

\section{INTRODUCTION}

Flagella are the structural and functional basis of the motility of Salmonella enterica. They are composed of three substructures: the basal body, the hook, and the filament. The basal body is the rotor of the flagella motor, embedded in the inner membrane; the hook is a flexible joint between the basal body and filament; and the filament, which is approximately 5$10 \mu \mathrm{m}$ long, acts as the propeller of the microbe (23). About 50 genes are associated with the structure, assembly, and function of the flagellum (17). However, the filament is constructed of only one kind of flagellin protein, which is synthesized in the cell and secreted from it through the central channel of the basic body and the hook of the flagellum and polymerizes automatically at the top of the filament. The filament of a peritrichous flagellum is composed of approximately 20,000 subunits of flagellin protein (1). All flagellar genes can be categorized into three classes based on their transcriptional regulation. Class 1 genes, including the master regulatory gene $f l h D C$, are necessary to activate the transcription of class 2 
genes. Some class 2 genes encode the proteins of the hook and basal body. A class 2 gene, fliA, encodes the flagellum-specific sigma factor FliA, which transcribes the class 3 genes involved in the motor and chemotaxis functions and filament structures $(13,18)$.

More than 90 kinds of flagellin have been identified in Salmonella by screening with the corresponding antisera (6). Flagellin genes are about 1500 bp in length, with two conservative terminal regions and a highly variable central region. The corresponding domain of flagellin is located on the surface of the filament and constitutes the flagellar epitope (20). Most S. enterica serovars are biphasic strains, which contain two different flagellin genes, designated fliC (phase I) and $f l j B$ (phase II), located at different loci on the chromosome (11). After treatment with anti-flagellin antiserum, the biphasic serovars can express FljB and FliC alternately, which is referred to as "phase variation". Salmonella enterica serovar Typhi is an enteric pathogen that causes systemic infections in humans, and is traditionally considered a monophasic strain, only containing $f l i C$, which encodes the $\mathrm{d}$ antigen or $\mathrm{j}$ antigen $(18,27)$.

In 1981, Guinee et al. first identified the H:z66 antigen in the $S$. enterica serovar Typhi strain, which was isolated from a patient who had traveled to Indonesia (8). Subsequent investigations show that z66-antigen-positive strains were only distributed in Indonesia and Southeast Asia. After it is induced with anti-z66 antiserum, the $z 66^{+}$strain can alter the expression of the flagellar antigen to the $d / j$ form, but the $d / j^{+}$strain never expresses the $z 66$ antigen after induction with anti-d/j antiserum (24). We previously identified the flagellin gene encoding the z66 antigen in $\mathrm{z}^{+} 6^{+}$strains as a $f l j B$-like gene (fljB:z66) (10). Recent research demonstrated that the fljB:z66 gene and fljA-like gene are located on a novel linear plasmid in the Typhi $z 66^{+}$strains (4). The promoter sequence of $f l j B: z 66$ is different from those of fliCs and fljBs of other biphasic strains (10). The regulation of $f l j B: z 66$ expression is as yet unclear.

To investigate the regulation of $f l j B: z 66$ expression in this study, we first generated deletion mutants of the flagellar regulator genes (fliA and $f l h D C$ ) and an $o m p R$ deletion mutant.
We examined the expression of $f l j B: z 66$ and $f l i C: j$ at the mRNA level and the secreted proteins in the wild-type $z 66^{+}$ strain and mutants at different osmolarities. We found that the expression of fljB:z66 is dependent on FliA and FlhDC, and that OmpR regulates the expression of $f l j B: z 66$ and affects the expression and secretion of FljB:z66 in different osmotic environments.

\section{MATERIALS AND METHODS}

\section{Bacterial strain and cultures}

A z66-antigen-positive wild-type strain of $S$. enterica serovar Typhi GIFU10007 was used in this study. The bacteria were grown with shaking at $37{ }^{\circ} \mathrm{C}$ in Luria-Bertani (LB) broth (pH 7.0) containing $50 \mathrm{mM}$ and $300 \mathrm{mM} \mathrm{NaCl}$, representing low- and high-osmolarity environments, respectively. The bacteria were grown overnight at different osmolarities, and then grown in fresh $\mathrm{LB}$ to $\log$ phase $\left(\mathrm{OD}_{600}\right.$ of 0.5$)$ at the same osmolarity to extract their RNA and secreted proteins. In this

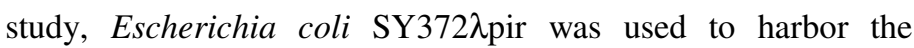
suicide plasmid pGMB151 for the construction of the targeted gene mutants.

\section{Construction of $f l h D C$, fliA, and ompR deletion mutants}

Mutants of $f l h D C, f l i A$, and $o m p R$ were generated by homologous recombination mediated by the suicide plasmid pGMB151. The primers used to prepare the recombinant DNAs and to investigate gene expression are listed in Table 1. To generate the $\operatorname{ompR}$ mutant, a $1.8-\mathrm{kb}$ fragment including $720 \mathrm{bp}$ of an ompR-homologous fragment was amplified with $\mathrm{PA} / \mathrm{B}$ from the wild-type strain and cloned into $E$. coli DH5 $\alpha$ with the pGEM®-T Easy vector, pGEM-T. A 297-bp fragment of the ompR gene (nucleotides 100-396) was deleted by treatment with restriction endonucleases $N s p \mathrm{~V}$ and BssHII and an exonuclease (TaKaRa, Japan). The fragment was then transferred into the suicide plasmid pGMB151 and transformed into E. coli SY372 $\lambda$ pir. The suicide plasmid carrying the deleted ompR gene was transferred into the wild-type strain by electroporation, as described previously (14). All plasmids were extracted with the 
QIAprep ${ }^{\circledR}$ Spin Miniprep Kit (Qiagen) in this study. After were amplified and ligated to form recombinant DNA fragments selective incubation on LB plates with ampicillin and lacking 471 bp of fliA and 689 bp of flhDC, as described in streptomycin, the bacteria were selectively incubated on LB Figure 1. These DNA fragments were then separately cloned into plates with $5 \%$ sucrose. The completely recombined strain was the BamHI site of the pGMB151 suicide plasmid. The positive selected by PCR, confirmed by sequencing analysis and defined suicide plasmids were then transformed separately into the target as the ompR deletion mutant. To generate the $f l h D C$ and fliA strain by electroporation. The selection and identification of the mutants, gene-specific upstream and downstream fragments mutants were performed as previously described (9).

Table 1. Primers used in this study.

\begin{tabular}{|c|c|c|}
\hline Primers & Sequence(5'-) & Purpose \\
\hline P-ompR-A (Bam HI) & TA $\underline{G G A T C C T C A G G C A C A A C G G T G T C G C A A}$ & \multirow{2}{*}{ ompR mutant construction } \\
\hline P-ompR-B $($ Bam $\mathrm{HI})$ & TA $\underline{G G A T C C G G A G C G G C G G T T A T G T T C T C A}$ & \\
\hline $\mathrm{P}-f l h D C-1 \mathrm{~A}(\mathrm{Bam} \mathrm{HI})$ & TAGGATCCATTATGTGATCTGCATCGCA & \multirow{4}{*}{ flhDC mutant construction } \\
\hline $\mathrm{P}-f l h D C-1 \mathrm{~B}(B g l \mathrm{II})$ & AA $\underline{A G A T C T A C T A A C T G G T T C G T C T C C G C ~}$ & \\
\hline $\mathrm{P}-f l h D C-2 \mathrm{~A}(B g l \mathrm{II})$ & TTAGATCTTTTGTTGAAAGTGGGTTGCT & \\
\hline P-flhDC-2B (Bam HI) & TGGATCCGCCAGTAAAATACCGAGGAA & \\
\hline $\mathrm{P}-f l i A-1 \mathrm{~A}($ Bam $\mathrm{HI})$ & TAGGATCCGGCGAAAAAACAATCATTCA & \multirow{4}{*}{ flliA mutant construction } \\
\hline P-fliA-1B $(B g l \mathrm{II})$ & AA $\underline{A G A T C T C C A T T A C A C C T T C A G C G G T A}$ & \\
\hline P-fliA-2A $(B g l \mathrm{II})$ & TT $\underline{A G A T C T C G T T A C A T C A A C T G C T G G A G ~}$ & \\
\hline P-fliA-2B (Bam HI) & TGGATCCATGTTTTGCTCGCTGAGGTA & \\
\hline P-fljB-1A $(S m a \mathrm{I})$ & TA $\underline{C C C G G G G G T A G A A G A T C A C T A C A A G A A}$ & \multirow{4}{*}{$\begin{array}{l}\text { pGMBfljB::lacZ } \\
\text { construction }\end{array}$} \\
\hline $\mathrm{P}-f l j B-1 \mathrm{~B}(K p n \mathrm{I})$ & GGGGTACCGAAGACTGAGATTTGTTCAG & \\
\hline $\mathrm{P}-f l j B-2 \mathrm{~A}(S a l \mathrm{I})$ & GTGTCGACGGTAATGGTACATATGAAGC & \\
\hline P-fljB-2B(SmaI $)$ & AA $\underline{C C C G G G G C G A C T T A C C T A C A T A C A T A}$ & \\
\hline P-flhD-sA & GAGATGGCAAACACACTGGG & \multirow{2}{*}{ qRT-PCR } \\
\hline P-flhD-sB & CCGTATCGTCCACTTCATTG & \\
\hline P-fliA-sA & ACCAACAACAGCCAACTTTT & \multirow{2}{*}{ qRT-PCR } \\
\hline P-fliA-Sb & ATTCAATCGCATCCATTACC & \\
\hline P-z66-sA & CAACCGCTAGTGATTTAGTTT & \multirow{2}{*}{ qRT-PCR } \\
\hline P-z66-sB & CTGTCCCTGTAGTAGCCGTAC & \\
\hline$P-j-s A$ & GAAACTGCTGTAACCGTTGA & \multirow{2}{*}{ qRT-PCR } \\
\hline$P-j-s B$ & CAACGCCAGTACCATCTGTA & \\
\hline P-lacZ-sA & CGTTACCCAACTTAATC & \multirow{2}{*}{ qRT-PCR } \\
\hline P-lacZ-sB & TGTGAGCGAGTAACAAC & \\
\hline $\mathrm{P}-g y r B-\mathrm{sA}$ & GAACAGCAGATGAACGAACT & \multirow{2}{*}{ qRT-PCR } \\
\hline $\mathrm{P}-g y r B-\mathrm{sB}$ & TTTTACCTTTCAGCGGCAGA & \\
\hline
\end{tabular}



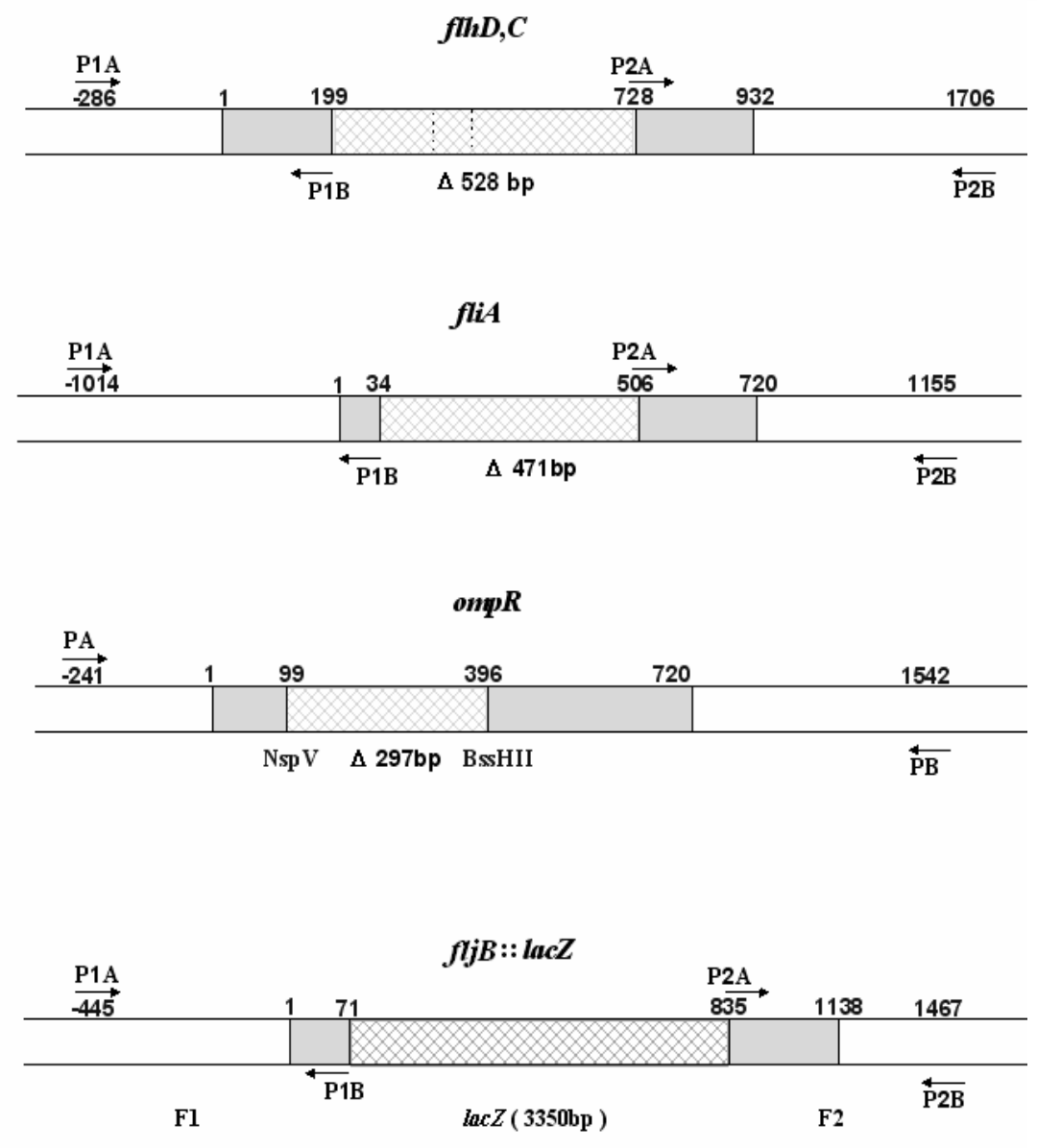

Figure 1. Primer design for construction of genedeleted mutants and the recombinant plasmid pGMB151(fljB::lacZ).

Specific primer pairs (P1A/B, P2A/B) located up- and down-stream of $f l h D C$ and fliA were designed to amplify homologic fragments that were then linked as the gene defective recombinant fragments. Primer pair P1A/B specific to the sequence up- and downstream of $o m p R$ were designed to amplify the homologic fragment, which was digested by $N s p \mathrm{~V}$ and BssHII and linked as the ompR defective recombinant fragment.

Primer pairs (P1A/B, P2A/B) specific to the sequence up- and downstream of $f l j B$ were designed to amplify two homologic fragments that were directionally linked with the promoterless lacZ cassette as the recombinant fragment. Recombinant fragments were individually cloned into the suicide plasmids for construction of gene-deleted mutants and the recombinant plasmid pGMB151(fljB::lacZ).

\section{RNA extraction and real-time quantitative reverse} transcription-polymerase chain reaction (qRT-PCR)

The bacterial cells were cooled on ice for $10 \mathrm{~min}$ and harvested by centrifugation $\left(4000 \mathrm{~g}\right.$ for $10 \mathrm{~min}$ at $4^{\circ} \mathrm{C}$ ). To destroy the cell envelope, the bacterial cells were resuspended in $100 \mu \mathrm{L}$ of lysozyme-TE buffer $(0.6 \mathrm{mg} / \mathrm{mL}$ lysozyme, $\mathrm{pH}$ 8.0), transferred into an NA extraction mini tube (AMR, Gifu, Japan), and vigorously shaken for $3 \mathrm{~min}$ at room temperature. Total RNA was then extracted with an RNeasy Mini Spin Column (Qiagen), according to the manufacturer's instructions. The quantity and quality of the extracted RNA were checked with an ND-1000 Spectrophotometer (NanoDrop Technologies, Wilmington, USA). To remove any traces of DNA, the extracted total RNA was treated with $1 \mathrm{U}$ of RNasefree DNase I (TaKaRa, Japan) at $37^{\circ} \mathrm{C}$ for $10 \mathrm{~min}$ and then incubated at $85^{\circ} \mathrm{C}$ for $15 \mathrm{~min}$ to inactivate the DNase.
Four pairs of primers specific for fljB:z66, fliC:j, flhD, or fliA were designed for qRT-PCR, and are shown in Table 1. Reverse transcription was primed with random octamer $\mathrm{N}_{8}$ and the SuperScript II kit (Invitrogen), was used according to the manufacturer's instructions. Each $20 \mu \mathrm{L}$ reaction contained 2 $\mu \mathrm{g}$ of total RNA and $10 \mathrm{nmol}$ of the random octamer. Each 1 $\mu \mathrm{L}$ of the reverse transcription product was subjected to a quantitative PCR assay, which was performed with primers Pz66A/B and PdA/B, as in the previous method (26). Fluorescence was measured at the end of the synthesis step in each cycle. Serially diluted plasmid DNAs encoding fljB:z66 were used to construct a standard curve at the same time as a reference from which to calculate the mRNA copy numbers in the samples. qRT-PCR was performed in duplicate for each RNA sample. The expression level of each gene was normalized by the expression level of $\operatorname{gyr} B$, which was 
assumed to be a steadily transcribed housekeeping gene in Salmonella (2). Data was expressed as the mean of expression ratio. After homogeneity of variance test, the Student's t-test was used to assess the statistical significance of differences between the groups.

\section{Sodium dodecyl sulfate-polyacrylamide gel electrophoresis} (SDS-PAGE) of secreted proteins and proteins in wholecell lysates and western blot analysis

The wild-type strain and the mutant strains were incubated overnight in $10 \mathrm{~mL}$ of $\mathrm{LB}$ broth containing $300 \mathrm{mM} \mathrm{NaCl}$ at 37 ${ }^{\circ} \mathrm{C}$. The secreted proteins were extracted as the previous method (10) and dissolved in $20 \mu \mathrm{L}$ of loading buffer. For the whole-cell lysates, mid-log phase (0.6 OD600) cells were harvested, resuspended in PBS. Equal volume of $2 \times$ loading buffer was added into each protein sample. After denaturizing at $100^{\circ} \mathrm{C}$ for 10 minutes, proteins were separated by SDS-PAG electrophoresis on $15 \%$ gels, and visualized with Coomassie Blue staining. The separated proteins were transferred by western blotting onto nitrocellulose membrane, which was probed with rabbit anti-z66 antiserum (National Institute of Infectious Disease, Japan) as the primary antibody and antirabbit Ig antibody conjugated to horseradish peroxidase (antirabbit Ig-Fc, AP Conjugate, Promega), as described previously(29).

\section{Transformation of wild type and ompR mutant with the recombinant plasmid pGMB151(fljB::lacZ) and $\beta$ - galactosidase assay}

To acquire a translational fusion of $f l j B:: l a c Z$, primer pairs P-fljB-1A/B and P-fljB-2A/B were used to amplify fragments F1 including whole promoter region of $f l j B: z 66$ and F2 located downstream from the $f l j B$ gene (Figure 1). A $S m a$ I site was added to the $5^{\prime}$ termini of primers $\mathrm{P}-f l j B-1 \mathrm{~A}$ and $\mathrm{P}-f l j B-2 \mathrm{~B}$, and a KpnI site and a SalI site were added to the $5^{\prime}$ termini of primers $\mathrm{P}-f l j B-1 \mathrm{~B}$ and $\mathrm{P}-f l j B-2 \mathrm{~A}$, respectively (Table 1$)$. The two fragments were amplified from the wild-type strain and digested with SalI and KpnI, respectively. To obtain the promoterless lac $Z$ cassette, the plasmid pSV- $\beta$-Galactosidase
Control Vector (Promega) was digested with KpnI and SalI. The lacZ cassette was purified with the Wizard ${ }^{\circledR}$ SV Gel and PCR Clean-up System (Promega), according to the manufacturer's instructions. After digestion with KpnI and SalI, fragments F1 and F2 were ligated with the promoterless lacZ cassette by T4 Ligase (TaKaRa, Japan) as the recombinant fragment, F1-lacZ-F2, which was then inserted into the BamHI site of plasmid pGMB151 to form a recombinant plasmid pGMBfljB::lacZ. The wild-type strain and the ompR mutant were transformed with the recombinant plasmid pGMBfljB::lacZ by electroporation. Transformed strains were grown in $\mathrm{LB}$ broth to an $\mathrm{OD}_{600}$ of $\sim 0.6$. When required, the media were supplemented with ampicillin $(100 \mu \mathrm{g} / \mathrm{mL})$. The $\beta$ galactosidase assays were performed as described previously (29). Each experiment was performed with three independent samples in duplicate and the values were recorded as $\beta$ galactosidase units (nanomoles per minute per $\mathrm{OD}_{650}$ unit per milliliter). The activity results were normalized to the plasmid copy numbers identified by qRT-PCR (lacZ gene).

\section{RESULTS}

\section{Expression of $f l j B: z 66$ and $f l i C$ in $o m p R, f l h D C$, and $f l i A$ mutants}

Flagellar gene expression is typically activated by the regulators FlhDC and FliA (16). The z66-positive S. enterica serovar Typhi is a special biphasic strain in which $f l j B: z 66$ is located on a linear plasmid, the source of which is unclear (5). To investigate whether $f l j B: z 66$ is regulated by FlhDC and FliA like other biphasic $S$. enterica, flhDC and fliA mutants were first prepared with a homologous recombination method mediated by a suicide plasmid. Sequence analysis demonstrated that the flhDC and fliA mutants were constructed successfully in this study. To show how flagellin expression is affected by the osmotic environment, an $\mathrm{ompR}$ mutant was also prepared. The expression of $f l j B: z 66$ and fliC in the ompR, flhDC, and fliA mutants at low and high osmolarity was analyzed by qRT-PCR with specific primers. The results are shown in Figure 2. The expression of fljB:z66 was higher at 
high osmolarity than at low osmolarity in the wild-type strain, and greatly reduced in the $f l h D C$ and fliA mutants at both high and low osmolarity. Compared with the wild-type strain, the expression of $f l j B: z 66$ in the ompR mutant was increased at low osmolarity but not at high osmolarity. In the ompR mutant, there was no obvious difference of $f l j B: z 66$ expression between incubating at low and high osmolarity. The expression of fliC was more than 10-fold lower than that of fljB:z66 in the wild- type and all mutant strains at both high and low osmolarity, whereas the patterns of expression were similar to that of $f l j B: z 66$.

These results indicate that the expression of $f l j B: z 66$ is dependent on the regulators FlhDC and FliA, like the expression of $f l i C$, and that the expression of $f l j B: z 66$ at low osmolarity is associated with the negative action of OmpR.
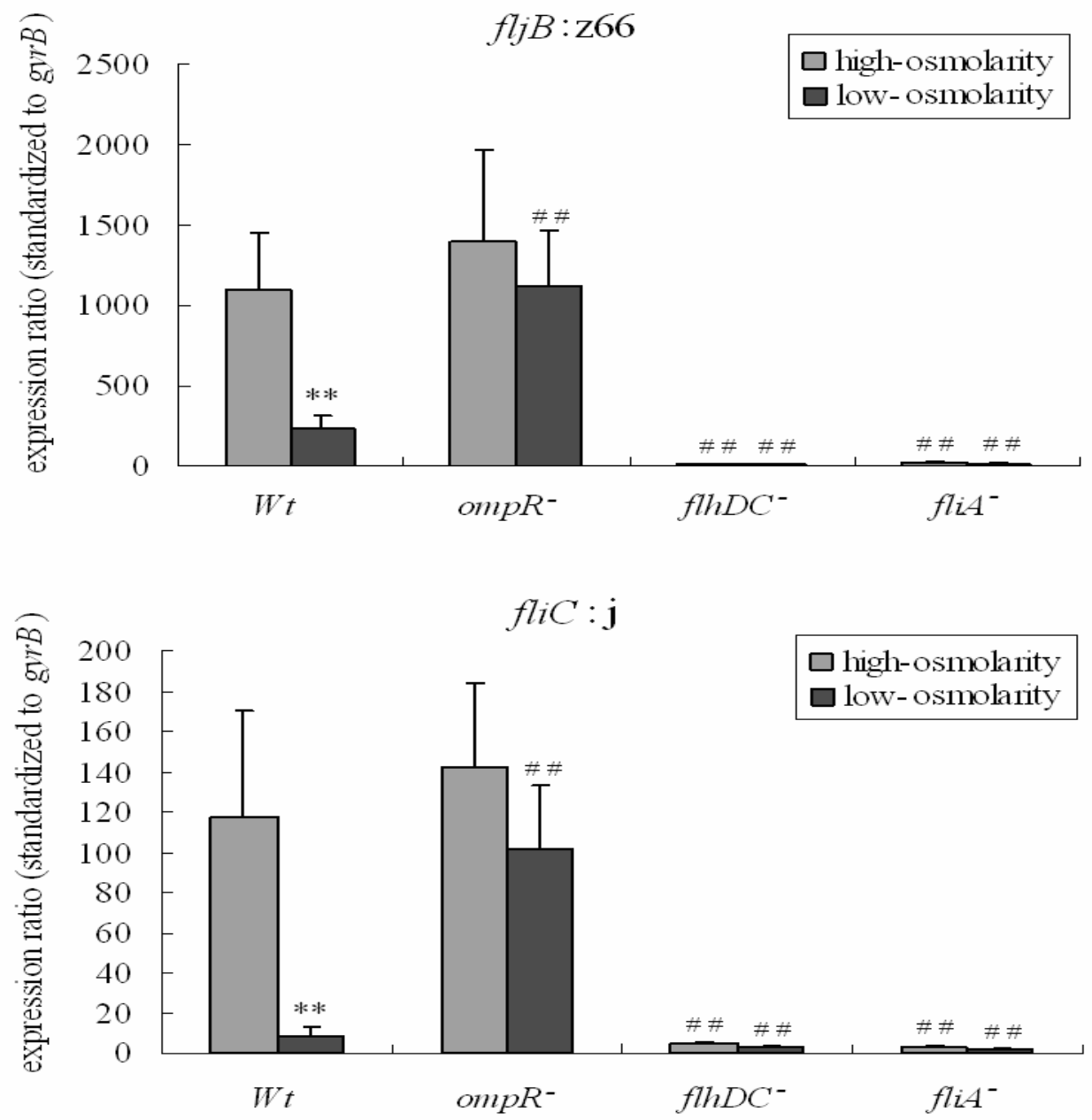

Figure 2. Expression of flagellin genes fljB:z66 and fliC in $o m p R, f l h D C$, and fliA mutants.

qRT-PCR was performed to investigate the expression of $f l j B: z 66$ and fliC in wild type strain (Wt), ompR mutant (ompR-), flhDC mutant ( $f l h D C$-) and fliA mutant (fliA-) incubated at high- and low-osmolarity. The expression of fljB:z66 and fliC was normalized with the expression of $g y r B$. Values reported represent means of three independent experiments carried out in duplicate. Bars represent standard deviations. Student's t-test was used to assess the statistical significance of differences between the groups. $* *$ $\mathrm{P}<0.01$ for a comparison with high-osmolarity in the same strain; ${ }^{\#} \mathrm{P}<0.01$ for a comparison with the wild-type strain at the same osmolarity. 


\section{Expression of $f l h D$ and $f l i A$ in the ompR mutant}

We previously found that the expression of the flagellar genes of Salmonella was strictly reduced early in the period of hyperosmotic stress and then increased after $2 \mathrm{~h}$ of stress (9, 26). Under stationary high-osmolarity conditions, the expression of $f l j B$ was higher than under low-osmolarity conditions, which seemed to be attributable to $\operatorname{OmpR}(9,26)$. To clarify whether the expression of $f l j B$ is regulated by OmpR through the regulators FlhDC and FliA in different osmotic conditions, the expression of $f l h D$ and fliA in the ompR mutant was investigated with qRT-PCR. The results are shown in Figure 3. The expression of $f l h D$ and fliA at low osmolarity was higher in the ompR mutant than in the wild-type strain, but there was no obvious difference under high-osmolarity conditions. These results suggest that the lower expression of $f l j B: z 66$ at low osmolarity is associated with the inhibition of the expression of flhDC and fliA by OmpR.
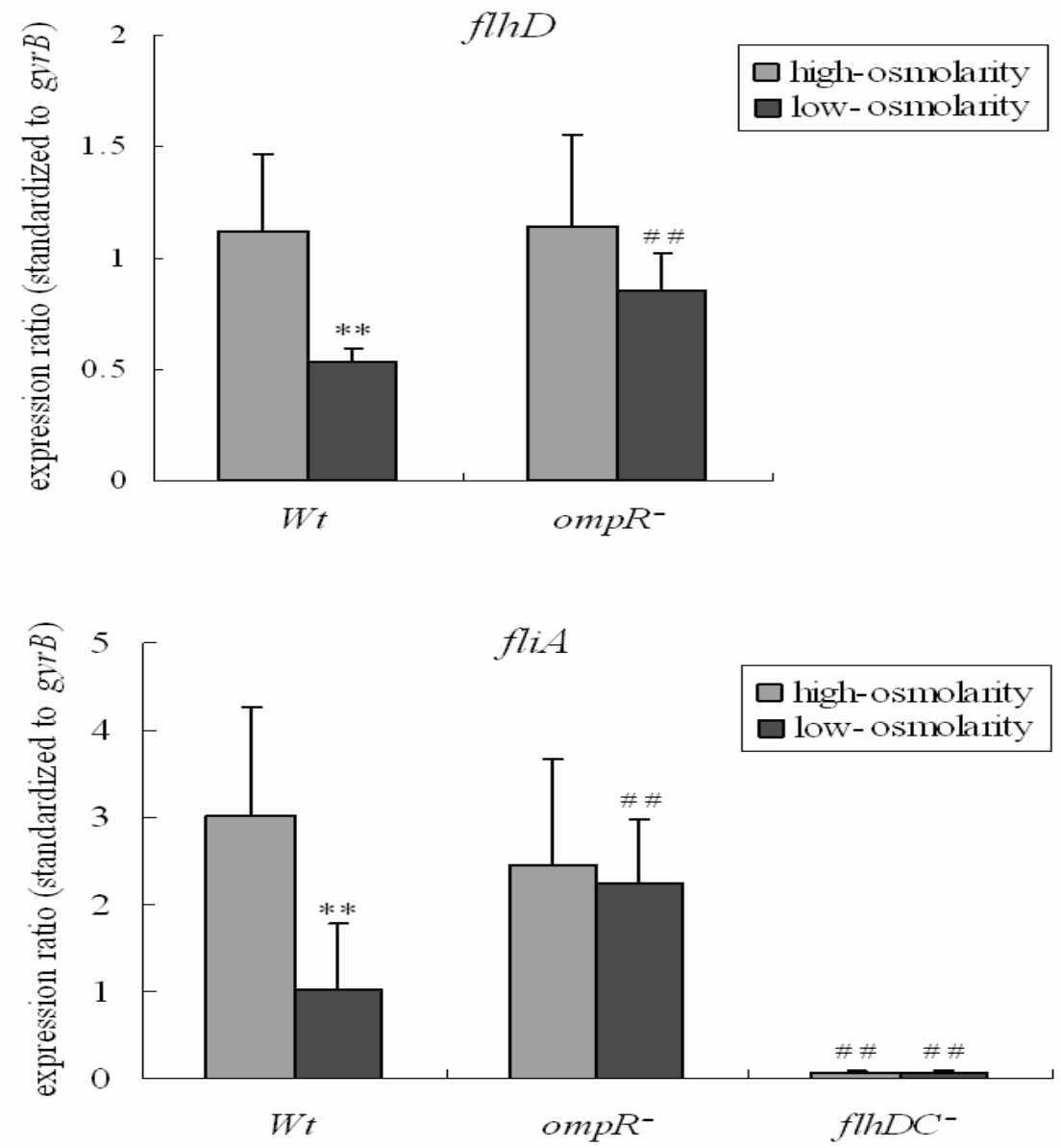

Figure 3. Expression of $f l h D$ and $f l i A$ in the ompR mutant.

Expression of $f l h D$ and fliA in the ompR mutant was investigated by qRT-PCR. Strains were incubated at high- and lowosmolarity. The expression of $f l h D$ and fliA was normalized with the expression of $g y r B$. Values reported represent means of three independent experiments carried out in duplicate. Bars represent standard deviations. Student's t-test was used to assess the statistical significance of differences between the groups. $* * \mathrm{P}<0.01$ for a comparison with high-osmolarity in the same strain; ${ }^{\# \#}$ $\mathrm{P}<0.01$ for a comparison with the wild-type strain in the same osmolarity. 
FljB:z66 in the secreted proteins of the ompR, $f l h D C$, and fliA mutants

Abundant flagellin monomers are synthesized in bacterial cells and secreted from the cells through the central channel of basic body and the hook of the flagellum, from which the flagellum is automatically polymerized (18). Flagellin in the bacterial secreted proteins may reflect the expression and secretion of bacterial flagellin $(10,28)$. In this study, the FljB:z66 in the secreted proteins was investigated by SDSPAGE and a western blotting assay. The results are shown in Figure 4. FljB:z66 was greatly reduced in the secreted proteins of both the flhDC and fliA mutants relative to that of the wildtype strain. These results are consistent with the observed fljB:z66 expression confirmed by qRT-PCR and discussed above. The amount of FljB:z66 in the secreted proteins of the wild-type strain was greater at high osmolarity than at low osmolarity, whereas there was no difference in the secreted proteins of the $\operatorname{ompR}$ mutant. There was also more FljB:z66 in the secreted proteins of the $\mathrm{ompR}$ mutant at low osmolarity than in the wild-type strain. It is likely that the expression and secretion of FljB:z66 is predominantly inhibited by OmpR at low osmolarity.

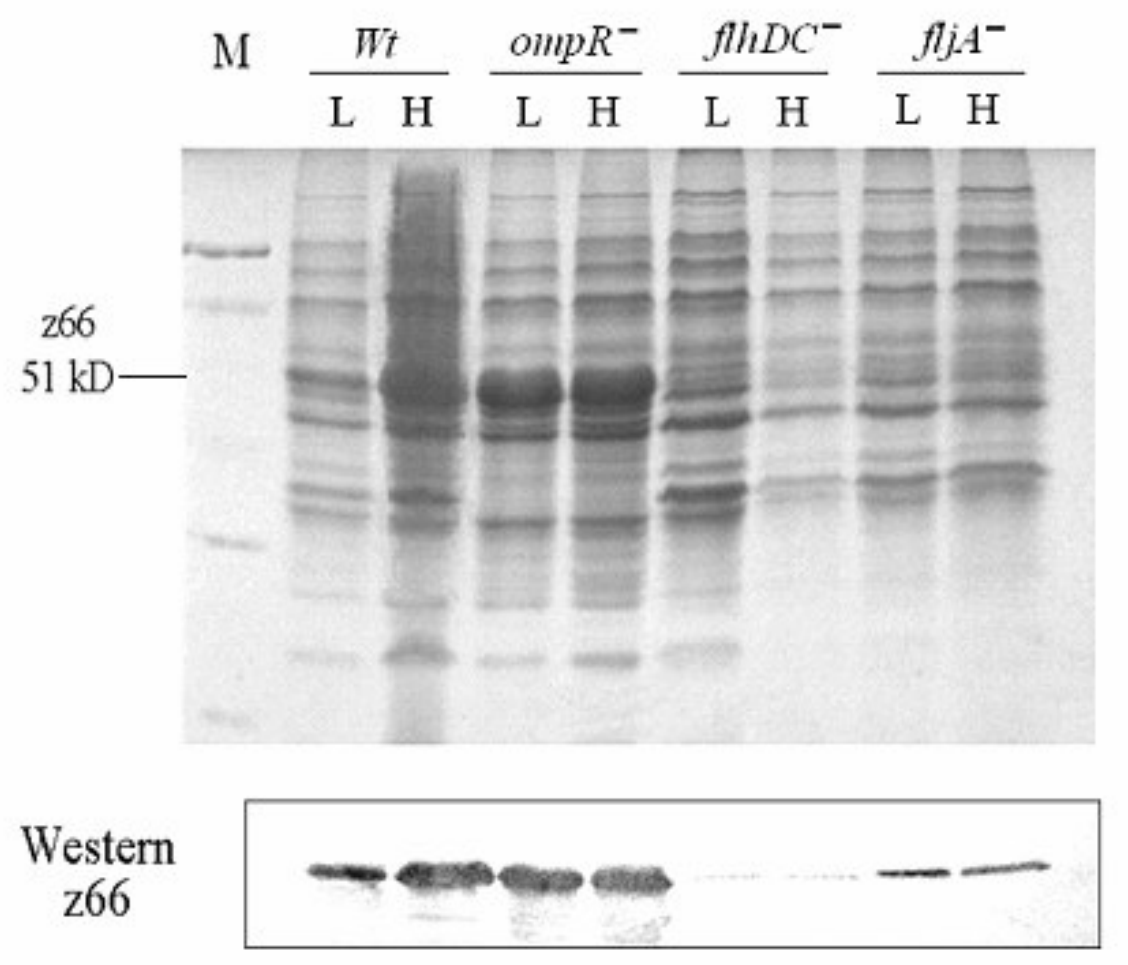

Figure 4. SDS-PAGE of secreted proteins and western blot probed with anti-z66 antibody.

Wild type strain and mutants were incubated in high $(\mathrm{H})$ and low $(\mathrm{L})$ osmotic conditions. Secreted proteins were extracted from cultures with trichloroacetic acid, separated by SDS-PAGE. Western blot was performed with anti-z66 antibody to reflect the amount of the flagellin FljB:z66 in secreted proteins. There was significantly more FljB:z66 in the secreted proteins of the wild-type strain at high osmolarity than at low osmolarity. In the ompR mutant, the amount of FljB:z66 in the secreted proteins did not differ significantly at high and low osmolarity. The amounts of FljB:z66 in the secreted proteins of the flhD and fliA mutants were greatly reduced compare with that in the wild-type strain. 


\section{Translational expression of $f l j B: z 66$ in the ompR mutant}

Vi polysaccharide synthesis in S. enterica serovar Typhi is promoted by OmpR, especially under low-osmolarity conditions (19). The Vi polysaccharides play a major role in protecting the pathogen against attack by host macrophages and are thought to act as a physical barrier to secretion in Salmonella $(3,19,28)$. To investigate whether the elevated levels of FljB:z66 in the proteins secreted by the ompR mutant are the result of overall increased secretion, the level of FljB:z66 inside the cells of the ompR mutant was investigated. The proteins in whole-cell lysates of Salmonella were harvested and the amount of FljB:z66 was analyzed by SDSPAG electrophoresis and western blotting. The results are shown in Figure 5A. FljB:z66 in the ompR mutant was lower than that in the wild-type strain in both high- and lowosmolarity environments.
To further investigate the translational expression of $f l j B: z 66$, the wild-type strain and the ompR mutant were transformed with a low-copy-number plasmid that contained a recombinant fragment of $f l j B$ inserted with a promoterless $l a c Z$ gene for a translational fusion. After incubation under low- and high-osmolarity conditions, the bacteria were harvested to analyze the activity of $\beta$-galactosidase. The results are shown in Figure 5B. The $\beta$-galactosidase activity of the ompR mutant was significantly lower than that in the wild-type strain at both low and high osmolarity. These results suggest that the translational expression of FljB:z66 in the ompR mutant is lower than that in the wild-type strain, which is similar to the previous western blot results for FljB:z66 in whole-cell lysates. These results revealed that $\mathrm{OmpR}$ might regulate translation of $f l j B: z 66$ in S. enterica serovar Typhi at different osmolarity.

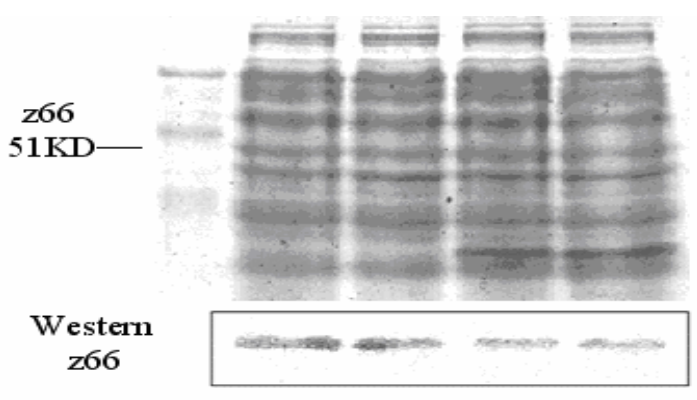

B

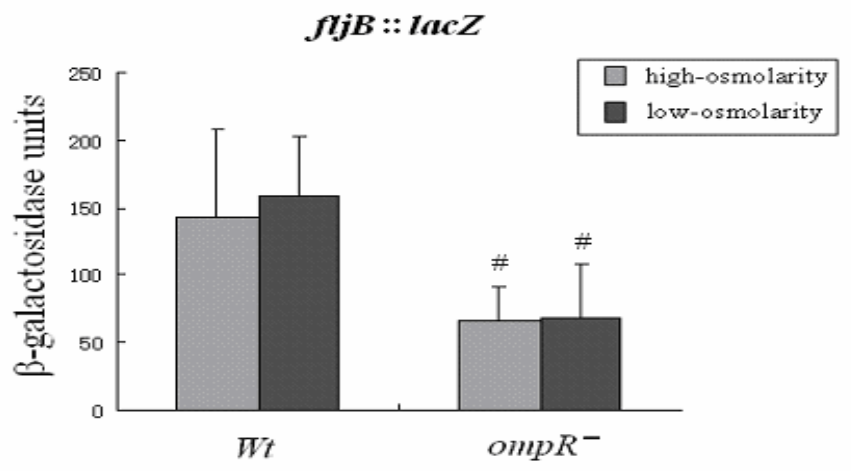

Figure 5. Translational expression of $f l j B: z 66$ in wild-type and $o m p R$ mutant strains.

A: Expression of FljB:z66 was investigated by SDS-PAG electrophoresis and western blotting in whole-cell lysates of the wild-type strain and $o m p R$ mutant incubated at high and low osmolarities. The expression of FljB:z66 in the ompR mutant was slightly lower than that in the wild-type strain at both low and high osmolarities. B: Assay of $\beta$-galactosidase activity in the wild-type and $o m p R$ mutant transformed with a recombinant plasmid was performed to reflect the translational expression of fljB:z66. The translational expression of $f l j B: z 66$ in the ompR mutant was lower than that in the wild-type strain $\left({ }^{\#} \mathrm{P}<0.05\right)$ at both low and high osmolarities, which is consistent with the results of western blotting. 


\section{DISCUSSION}

The motility of S. enterica serovar Typhi depends on the structure and function of the flagellum. The z66-positive $S$. enterica serovar Typhi is a special biphasic strain containing a $f l j B A$-like gene on a linear plasmid, which encodes the flagellin z66 antigen and a fliC repressor $(4,29)$. The expression and regulation of $f l j B: z 66$ is interesting because the promoter of $f l j B: z 66$ is different from the promoters of the $f l j B s$ of other biphasic $S$. enterica (10). Whether the $f l j B: z 66$ gene is a class 3 flagellar gene has not been clarified.

To investigate the characteristics of the regulation of $f l j B: z 66$ gene expression in this study, we constructed mutants flhDC $\mathrm{C}^{-}$and $\mathrm{fliA}^{-}$in which the flagellar-regulator genes were deleted. With qRT-PCR, we first found that $f l j B: z 66$ gene expression is dependent on the regulator genes flhDC and fliA. This was confirmed with a western blotting assay of FljB:z66 in the bacterially secreted proteins. The cascade of flagellin expressional regulation in Enterobacteriaceae are well known as that the central regulator $F \mathrm{Fh}_{2} \mathrm{C}_{2}$ activates transcription of class 2 flagellar genes and fliA, which then promotes expression of FliC and other class 3 flagellin genes. Therefore, we believe that the $f l j B: z 66$ gene is also a class 3 flagellar gene in S. enterica serovar Typhi.

The flagellum is an important pathogenic factor of $S$. enterica serovar Typhi $(12,21)$. During the invasion process, the pathogen encounters osmotic upshift stress in the small intestinal tract. We previously found that the expression of $f l j B: z 66$ is clearly reduced in the early stage of osmotic stress and increases under stationary high-osmolarity conditions (9, 26). OmpR is an osmoresponsive regulator, which forms a twocomponent regulatory system with EnvZ, an osmosensor located on the inner membrane of the cell (7). The previous research found that phosphorylated OmpR was a negative regulator for $f l h D$ expression in E. coli in the high osmolarity condition (22). Another research found that inactivation of ompR promoted flhDC expression and swarming in Xenorhabdus nematophila incubated on LB plate with middle osmolarity (15). However, inactivation of $\mathrm{ompR}$ did not affect
flhDC expression in S. enterica serovar Typhimurium (16). The relationship between OmpR and the expression of fljB:z66 was first investigated in this study. After the preparation of an ompR mutant, the expression of fljB:z66 was analyzed by qRTPCR. The results showed that OmpR inhibited the transcription of $f l j B: z 66$ at low osmolarity but not at high osmolarity. This also occurs in the expression of the phase-1 flagellin gene $f l i C:$ j. Combined with the results of $f l h D$ and fliA expression assay, we consider that nonphosphorylated OmpR may inhibit the expression of $f l j B: z 66$ indirectly by repressing the expression of flhDC in S. enterica serovar Typhi under lowosmolarity conditions. To investigate whether the inhibition of $f l h D C$ is direct, we performed the gel shift experiment with an

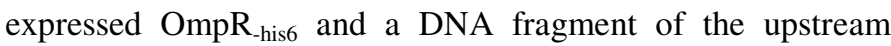
region of the $f l h D(-286$ to $+199 \mathrm{bp})$. We did not find the positive result comparing with a positive control with the upstream region of the ompF (data not shown). So, we speculate that $\mathrm{OmpR}$ may indirectly regulate the transcription of $f l h D C$.

Flagellin monomers are secreted by the bacterium and are easily detected among the secreted proteins. Experiments have suggested that Vi capsular antigen is a barrier to the secretion of flagellin in S. enterica serovar Typhi (3). Other research has demonstrated that TviA, an activator of Vi capsular antigen, reduces flagellin secretion (25). Vi capsular antigen is a negative factor for $S$. enterica serovar Typhi invasion into host enteric $M$ cells. When entering a high-osmolarity enteric environment, $S$. enterica serovar Typhi can increase its expression and secretion of flagellin and SPI-1 Sip proteins by reducing the expression of Vi capsular genes (3). In this study, we also found that large amounts of FljB:z66 appeared in the secreted proteins at high osmolarity. In ompR mutant amounts of FljB:z66 appeared in the secreted proteins at low osmolarity almost same as that at high osmolarity. However, translational expression investigation of fljB:z66 revealed that OmpR did not repressed the synthesis of FljB:z66 in S. enterica serovar Typhi both at high and low osmolarity. Large amount of FljB:z66 in secreted protein in $o m p R$ mutant at low osmolarity was predominantly the result of the increase in secretion by $S$. 
enterica serovar Typhi. It is interesting that the translational expression of $f l j B: z 66$ was not significantly different under high and low osmotic conditions, although its transcriptional expression differed. It is likely that regulation of FljB:z66 secretion is major response to osmolarity and expressional regulation of fljB:z66 by OmpR is happen not only in transcriptional level but also in some posttranscriptional levels in S. enterica serovar Typhi at different osmolarity.

\section{ACKNOWLEDGMENTS}

This work was supported by grants from National Natural Science Foundation of China (30570088), National Special Scientific Program (2008ZX10004-009), and SIT of Jiangsu University (2008-018-02).

\section{REFERENCES}

1. Aizawa, S.I. (1996). Flagellar assembly in Salmonella typhimurium. Mol. Microbiol. 19(1), 1-5.

2. Ansong, C.; Yoon, H.; Porwollik, S.; Mottaz-Brewer, H.; Petritis, B.O.; Jaitly, N.; Adkins, J.N.; McClelland, M.; Heffron, F.; Smith, R.D. (2009). Global systems-level analysis of $\mathrm{Hfq}$ and $\mathrm{SmpB}$ deletion mutants in Salmonella: implications for virulence and global protein translation. PLoS. One. 4(3), e4809.

3. Arricau, N.; Hermant, D.; Waxin, H.; Ecobichon, C.; Duffey, P.S.; Popoff, M.Y. (1998). The RcsB-RcsC regulatory system of Salmonella typhi differentially modulates the expression of invasion proteins, flagellin and Vi antigen in response to osmolarity. Mol. Microbiol. 29(3), $835-850$.

4. Baker, S.; Holt, K.; Whitehead, S.; Goodhead, I.; Perkins, T.; Stocker, B.; Hardy, J.; Dougan, G. (2007). A linear plasmid truncation induces unidirectional flagellar phase change in H:z66 positive Salmonella Typhi. Mol. Microbiol. 66(5), 1207-1218.

5. Baker, S.; Hardy, J.; Sanderson, K.E.; Quail, M.; Goodhead, I.; Kingsley, R.A.; Parkhill, J.; Stocker, B.; Dougan, G. (2007). A novel linear plasmid mediates flagellar variation in Salmonella Typhi. PLoS. Pathog. 3(5), e59.

6. Edwards, P.R.; Ewing, W.H. (1986). Edwards and Ewing's Identification of Enterobacteriaceae. Elsevier Science Publishing Co., New York.

7. Gibson, M.M.; Ellis, E.M.; Graeme-Cook, K.A.; Higgins, C.F. (1987). OmpR and EnvZ are pleiotropic regulatory proteins: positive regulation of the tripeptide permease (tppB) of Salmonella typhimurium. Mol. Gen. Genet. 207(1), 120-129.

8. Guinee, P.A.; Jansen, W.H.; Maas, H.M.; Le Minor, L.; Beaud, R.
(1981). An unusual H antigen (Z66) in strains of Salmonella typhi. Ann. Microbiol (Paris). 132(3), 331-334.

9. Huang, X.; Xu, H.; Sun, X.; Ohkusu, K.; Kawamura, Y.; Ezaki, T. (2007). Genome-Wide Scan of the Gene Expression Kinetics of Salmonella enterica Serovar Typhi during Hyperosmotic Stress. International Journal of Molecular Sciences. 8(2), 116-135.

10. Huang, X.; Phung le, V.; Dejsirilert, S.; Tishyadhigama, P.; Li, Y.; Liu, H.; Hirose, K.; Kawamura, Y.; Ezaki, T. (2004). Cloning and characterization of the gene encoding the Z66 antigen of Salmonella enterica serovar Typhi. FEMS. Microbiol. Lett. 234(2), 239-246.

11. Ikeda, J.S.; Schmitt, C.K.; Darnell, S.C.; Watson, P.R.; Bispham, J.; Wallis, T.S.; Weinstein, D.L.; Metcalf, E.S.; Adams, P.; O'Connor, C.D.; O'Brien, A.D. (2001). Flagellar phase variation of Salmonella enterica serovar Typhimurium contributes to virulence in the murine typhoid infection model but does not influence Salmonella-induced enteropathogenesis. Infect. Immun. 69(5), 3021-3030.

12. Jones, B.D.; Lee, C.A.; Falkow, S. (1992). Invasion by Salmonella typhimurium is affected by the direction of flagellar rotation. Infect. Immun. 60(6), 2475-2480.

13. Kerridge, D.; Horne, R.W.; Glauert, A.M. (1962). Structural components of flagella from Salmonella typhimurium. J Mol. Biol. 4, 227-238.

14. Khan, A.Q.; Zhao, L.; Hirose, K.; Miyake, M.; Li, T.; Hashimoto, Y.; Kawamura, Y.; Ezaki, T. (1998). Salmonella typhi rpoS mutant is less cytotoxic than the parent strain but survives inside resting THP-1 macrophages. FEMS. Microbiol. Lett. 161(1), 201-208.

15. Kim, D.J.; Boylan, B.; George, N.; Forst, S. (2003). Inactivation of ompR promotes precocious swarming and $f l h D C$ expression in Xenorhabdus nematophila. J. Bacteriol. 185(17), 5290-5294.

16. Kutsukake, K. (1997). Autogenous and global control of the flagellar master operon, flhD, in Salmonella typhimurium. Mol. Gen. Genet. 254(4), 440-448.

17. Kutsukake, K.; Ohya, Y.; Iino, T. (1990). Transcriptional analysis of the flagellar regulon of Salmonella typhimurium. J. Bacteriol. 172(2), 741747.

18. Macnab, R.M. (1996). Flagella and motility. In:Neidhardt, F. C.; Curtiss, R. I.; Ingraham, J. L.; Lin, E. C. C.; Low, K. B.; Magasanik, B.; Reznikoff, W. S.; Riley, M.; Schaechter, M.; Umbarger, H. E.(eds). Escherichia coli and Salmonella : cellular and molecular biology. American Society for Microbiology, Washington, D.C., p.123-141.

19. Pickard, D.; Li, J.; Roberts, M.; Maskell, D.; Hone, D.; Levine, M.; Dougan, G.; Chatfield, S. (1994). Characterization of defined ompR mutants of Salmonella typhi: ompR is involved in the regulation of $\mathrm{Vi}$ polysaccharide expression. Infect. Immun. 62(9), 3984-3993.

20. Popoff, M.Y.; Minor, L.L. (1992). Antigenic formulas of the Salmonella serovars, 6th revision. WHO Collaborating Center for Reference and Research on Salmonella. Institute Pasteur, Paris, France,

21. Schmitt, C.K.; Ikeda, J.S.; Darnell, S.C.; Watson, P.R.; Bispham, J.; Wallis, T.S.; Weinstein, D.L.; Metcalf, E.S.; O'Brien, A.D. (2001). Absence of all components of the flagellar export and synthesis 
machinery differentially alters virulence of Salmonella enterica serovar Typhimurium in models of typhoid fever, survival in macrophages, tissue culture invasiveness, and calf enterocolitis. Infect. Immun. 69(9), 56195625 .

22. Shin, S.; Park, C. (1995). Modulation of flagellar expression in Escherichia coli by acetyl phosphate and the osmoregulator OmpR. J. Bacteriol. 177(16), 4696-4702.

23. Sosinsky, G.E.; Francis, N.R.; Stallmeyer, M.J.; DeRosier, D.J. (1992). Substructure of the flagellar basal body of Salmonella typhimurium. J. Mol. Biol. 223(1), 171-184.

24. Tamura, K.; Sakazaki, R.; Kuramochi, S.; Nakamura, A. (1988). Occurrence of H-antigen Z66 of R phase in cultures of Salmonella serovar typhi originated from Indonesia. Epidemiol. Infect. 101(2), 311314.

25. Winter, S.E.; Raffatellu, M.; Wilson, R.P.; Russmann, H.; Baumler, A.J. (2008). The Salmonella enterica serotype Typhi regulator TviA reduces interleukin- 8 production in intestinal epithelial cells by repressing flagellin secretion. Cell. Microbiol. 10(1), 247-261.

26. Xu, S.; Zhang, H.; Sheng, X.; Xu, H.; Huang, X. (2008). Transcriptional expression of $f l j B: z 66$, a flagellin gene located on a novel linear plasmid of Salmonella enterica serovar Typhi under environmental stresses. New. Microbiol. 31(2), 241-247.

27. Yamaguchi, S.; Fujita, H.; Sugata, K.; Taira, T.; Iino, T. (1984). Genetic analysis of $\mathrm{H} 2$, the structural gene for phase-2 flagellin in Salmonella. $J$. Gen. Microbiol. 130(2), 255-265.

28. Zhao, L.; Ezak, T.; Li, Z.Y.; Kawamura, Y.; Hirose, K.; Watanabe, H. (2001). Vi-Suppressed wild strain Salmonella typhi cultured in high osmolarity is hyperinvasive toward epithelial cells and destructive of Peyer's patches. Microbiol. Immunol. 45(2), 149-158.

29. Zou, X.; Huang, X.; Xu, S.; Zhou, L.; Sheng, X.; Zhang, H.; Xu, H.; Ezaki, T. (2009). Identification of a fljA gene on a linear plasmid as the repressor gene of fliC in Salmonella enterica serovar Typhi. Microbiol. Immunol. 53(4), 191-197. 